\title{
Culture patterns and sorting of rat Sertoli cell secretory proteins
}

\author{
HIROSHI UEDA ${ }^{1,2}$, LAURA L. TRES ${ }^{2,3}$ and ABRAHAM L. KIERSZENBAUM ${ }^{1,2, *}$ \\ 'Department of Cell Biology and Anatomy ${ }^{2}$ Department of Pediatncs and ${ }^{3}$ The Laboratories for Reproductive Biologv. School of Medicine, \\ Unversity of North Carolma at Chapel Hill, NC 27599, USA \\ - Author for correspondence at Department of Cell Bıology and Anatomy, C.B.\# 7090, 348 Swing Building, Chapel Hill, NC 27599, USA
}

\section{Summary}

A cocultivation chamber and two types of permeable substrates have been used to study: (1) the culture patterns of rat Sertoli and peritubular cells, and Sertoli cells cocultured with spermatogenic cells or peritubular cells; and (2) the polarized secretion of Sertoli cell-specific proteins transferrin, S70 and S45-S35 heterodimeric protein. Substrates included a nylon mesh (with openings of $100 \mu \mathrm{m}$ ) coated with extracellular matrix (ECM) material and an uncoated microporous filter (with pores of $0.45 \mu \mathrm{m}$ ). Sertoli cells cultured on ECM-coated nylon mesh organized a continuous sheet of multilayered epithelial cells essentially devoid of spermatogenic cells while peritubular cells formed a layer of squamous cells. Sertoli cells cultured on uncoated microporous substrate formed a continuous sheet of cuboidal epithelial cells with numerous basal cytoplasmic processes projecting into the substrate and abundant apically located spermatogenic cells, while peritubular cells organized one or two layers of loose squamous cells. $\left[{ }^{35} \mathrm{~S}\right]$ methionine-labelled secretory proteins resolved by two-dimensional polyacrylamide gel electrophoresis and autoradiography displayed cell-specific patterns that were slightly influenced by the type of substrate. Sertoli cells cocultured with peritubular cells on uncoated microporous substrate under conditions that enabled separation of apical and basal surfaces, secreted proteins in a polarized fashion. While transferrin was released bidirectionally, S45-S35 heterodimeric protein was released apically. S70 was detected in both apical and basal compartments. We conclude from these studies that: (1) the number of spermatogenic cells decreases when Sertoli-spermatogenic cell cocultures are prepared on ECM-coated nylon substrate; and (2) Sertoli cells in coculture with spermatogenic or peritubular cells on uncoated microporous substrate, organize continuous sheets displaying polarized protein secretion.

Key words: Sertoli cells, spermatogenic cells, peritubular cells, cell cocultivation chamber.

\section{Introduction}

Sertoli and peritubular cells contribute to the formation of a selective permeability barrier responsible for the maintenance of a unique environment within the seminiferous tubule. This barrier is bidirectional because it limits access of plasma-borne molecules to components of the seminiferous epithelium and restricts the release of spermatogenic cell antigens and, presumably, other substances into the general circulation (Setchell, 1978). Sertoli cells, in particular, are specialized to perform vectorial functions, since they

Journal of Cell Science 89, 175-188 (1988)

Printed in Great Britain (C) The Company of Biologists Limited 1988 bridge the basally located blood supply space with the apically located seminiferous tubular lumen.

Experimental difficulties involved in the manipulation of the intact testis have been overcome in part by the use of in vitro systems such as cultured testicular cells and short-term incubated seminiferous tubules (Kierszenbaum et al. 1987). Rat Sertoli and peritubular cells placed separately in cell culture synthesize and secrete proteins that are characteristic of each cell type (DePhilip \& Kierszenbaum, 1982; Kissinger et al. 1982), and also common to each other (Kierszenbaum et al. 1986a). Sertoli and peritubular cells synthesize different extracellular matrix components (Tung et al. 
1985; Skinner et al. 1985). It has been suggested that these two cells cooperate in the production and formation of the seminiferous tubular basal lamina (Tung $e t$ al. 1985). In addition, media conditioned by cultured peritubular cells have a paracrine effect on cultured Sertoli cells, demonstrated by increased secretion of androgen-binding protein (Tung \& Fritz, 1980; Hutson \& Stocco, 1981; Hutson, 1983; Skinner \& Fritz, 1985) and transferrin (Skinner \& Fritz, 1985).

Studies concerning cell polarity of transporting epithelia have been stimulated by growing epithelial cells on permeable supports, such as nitrocellulose filters (Misfeldt et al. 1976) or collagen-coated nylon mesh (Cereijido et al. 1978), to facilitate basal nutrient uptake by cells. Rat Sertoli cells cultured on a microporous substrate coated with an extracellular matrix extract become highly polarized and the secretion of androgen-binding protein and transferrin is enhanced (Hadley et al. 1985). Similarly, polarized Sertoli cells cultured on an uncoated microporous substrate release androgen-binding protein and transferrin into apical and basal cell compartments (Janecki \& Steinberger, 1986). However, a unidirectional apical sorting of transferrin by cultured Sertoli cells has been reported involving a transcellular mechanism for the translocation of iron from plasma transferrin to testicular transferrin (Djakiew et al. 1986). The idea of a polarized function for Sertoli cells cultured on a microporous substrate is supported by the apical arrangement of differentiating spermatogenic cells in coculture with rat Sertoli cells (Tres \& Kierszenbaum, 1986; Kierszenbaum \& Tres, 1987).

While available experimental data indicate that cultured Sertoli and peritubular cells can maintain structural and functional interaction, and Sertoli cells can direct their secretory proteins in a vectorial fashion, little is known about the vectorial sorting of two Sertoli cell secretory proteins, designated $\mathrm{S} 70$ and $\mathrm{S} 45-\mathrm{S} 35$ heterodimeric protein (Kierszenbaum et al. 1986a, 1987), which are known to be temporally and cyclically expressed during spermatogenesis (Shabanowitz et al. 1986).

In this paper, we evaluate the growth patterns of rat Sertoli and peritubular cells on two different permeable substrates developed for automated cell-perifusion studies (Tres \& Kierszenbaum, 1986; Kierszenbaum \& Tres, 1987) and the vectorial sorting of transferrin, S70 and S45-S35 heterodimeric protein by rat Sertoli cells in coculture with spermatogenic or peritubular cells. We report: (1) the bidirectional release of radiolabelled transferrin and $\mathrm{S} 70 ;(2)$ the unidirectional release of S45-S35 heterodimeric protein; and (3) the insignifcant contribution of cocultured cells to the polarized secretion of Sertoli cell-specific proteins.

\section{Materlals and methods}

\section{Isolation and culture of rat Sertoli and peritubular \\ cells}

Sertoli cell cultures were prepared from 20- to 22-day-old rats (Charles River Laboratories, Wilmington, MA) as described (Kierszenbaum \& Tres, 1981; Kierszenbaum et al. 1986a). A detailed protocol for the preparation of cultured peritubular cells has been reported (Kierszenbaum et al. 1986b).

\section{Preparation of cell cultures on extracellular matrix- coated and uncoated substrates}

Two types of substrates were used: a nylon mesh and a microporous filter. While the coated nylon mesh exposes wide and unobstructed basal cell surface areas to access by culture medium (openings about $100 \mu \mathrm{m}$ wide), the exposed cell surface area provided by the microporous filter is more limited (pores of $0.45 \mu \mathrm{m}$ ). Cells were plated on sterile discs (22 $\mathrm{mm}$ in diameter) of Nitex screening fabric (nylon monofilament HC 3-110, mesh opening: $103 \mu \mathrm{m}, 40 \%$ open area, TETKO, Inc., Elmsford, $\mathrm{NY}^{\mathrm{Y}}$ ) coated with an extracellular matrix extract (ECM) (a gift from Dr Hynda $\mathrm{K}$. Kleinman) or on sterile uncoated HATF filter discs $(0.45 \mu \mathrm{m}$ pores, Millipore, Bedford, MA). Undiluted ECM was deposited on nylon discs (kept in $35 \mathrm{~mm}$ plastic culture dishes) forming a $1 \mathrm{~mm}$ thick gel layer. In other experiments, ECM was diluted 1:1 (v/v) with Hanks' Balanced Salt Solution (HBSS), and two coats of diluted ECM were placed on nylon discs. ECMcoated nylon discs were rinsed during $24 \mathrm{~h}$ with several changes of HBSS to remove traces of salt and urea from the ECM extract.

Discs were inserted within sterilized teflon cylinders (labelled a in Fig. 1). An inner rim in the cylinder provided support and spacing to the disc (Fig. 1). A teflon insert (labelled $b$ in Fig. 1) kept the disc flat and in place. The teflon insert has two small holes for insertion of stainless steel forceps that facilitate assembly and removal of the teflon insert.

Sertoli cells (associated with about $30 \%$ spermatogonia and meiotic prophase spermatocytes) were resuspended in Eagle's minimum essential medium (EMEM) supplemented with $10 \%$ foetal bovine serum (Sterile Systems, Logan, UT) and plated directly on ECM-coated nylon or uncoated HATF discs housed in the teflon holder. Peritubular cells were cultured in culture flasks in serum-supplemented ENEM as reported (Kierszenbaum et al. 1986b), detached with $0.25 \%$ trypsin in HBSS and plated on ECM-coated nylon or uncoated HATF discs that were previously assembled in the teflon holder. Specimens were placed in either $35 \mathrm{~mm}$ culture dishes or six-well plates. After $24 \mathrm{~h}$, serum-supplemented medium was removed from Sertoli cell cultures, replaced with serum-free EMEM supplemented with hormones and growth factors (TKM; Tres et al. 1986), and replenished every $24 \mathrm{~h}$ for 6 consecutive days. Peritubular cells were maintained in serum-supplemented EMEM. Nylon discs were examined for cell confluency by phase-contrast microscopy. Because of the translucent nature of HATF discs, cell confluency was monitored using a stereomicroscope equipped with zoom lenses that allowed visualization of the cell culture surface. Possible cross-contamination of Sertoli 

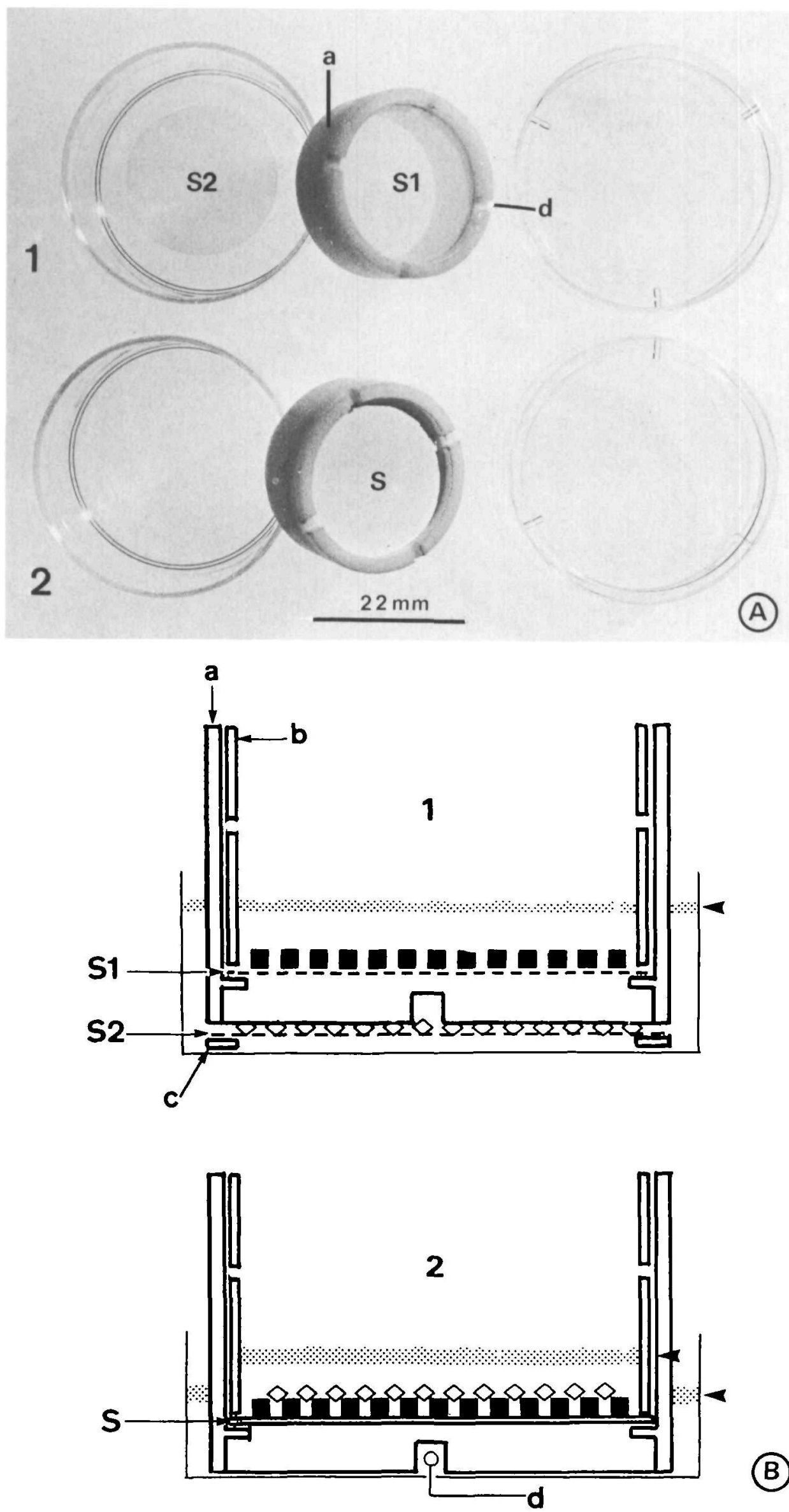

Fig. 1. Photograph (A) and diagrammatic representation (B) of the one- and twocompartment cultivation and cocultivation device. A. Part 1 illustrates a teflon cylinder (a) with grooves (d) at the basal edge. $\mathrm{Sl}$ in the cylinder is translucent nylon mesh coated with extracellular matrix extract. S2 in the bottom part of a $35-\mathrm{mm}$ culture dish is a simılar nylon-ECM-coated substrate with a different cell population than in S1. Part 2 illustrates an identical teflon cylinder with an assembled, non-transparent HATF filter (S). B. Part 1 is a diagrammatic representation of part 1 in $A$; $a$ is the teflon cylinder; $b$ is the insert to maintain the substrate in place. S1 within the cylinder is a nylon-ECM-coated substrate with a cell population that is different from S2 placed under the cylnder for cocultivation using a one-compartment cell-cell interacting mode. $\mathrm{S} 2$ is placed on a flat teflon spacer (c) to permit access of medium to basal cell surfaces. The distance between $\mathrm{S} 1$ and $\mathrm{S} 2$ is $2 \mathrm{~mm}$. Part 2 is a diagram of part 2 in $A . S$ is an uncoated HATF microporous substrate containing a cell coculture $(\boldsymbol{\square}, \triangle) ; \mathrm{d}$ is one of four grooves permitting medium access to basal cell surfaces. Arrowheads in 1 and 2 indicate fluid levels in each compartment. Diagrams are not to scale. 
cell cultures with peritubular cells was evaluated by immunofluorescence microscopy of representative samples using monoclonal antıbody IFP-SC as reported (Kierszenbaum et al. 1986b), and by microscopic examination of plasticembedded specimens (see below). Cross-contamination was less than $1 \%$.

\section{Cell-cell interaction}

Two types of cell interaction studies were carried out using cocultivation chambers containing either ECM-coated nylon or uncoated HATF substrates (Fig. 1). In the first type of cell interaction, Sertolı or peritubular cells prepared on ECMcoated nylon substrates were allowed to interact functionally with each other, but direct cell contact was avoided. This cell-cell interaction between Sertoli and peritubular cells resulted in the combination of apically and basally sorted cell products within a one-flud compartment (Fig. 1, part 1). The second type of cell interaction was generated by allowing populations of Sertoli and peritubular cells to establish direct contact with each other when plated on uncoated HATF microporous substrates. Both the microporous substrate and the continuous nature of the Sertoli cell monolayer resulted in an apical/basal-compartmentalized fluid chamber in which apically and basally sorted cell products could be identified (Figs 1, 2).

One-fluid comparlment. Interaction between Sertoli and peritubular cells was accomplished using two ECM-coated nylon discs, each containing Sertoli or peritubular cells. Reciprocal interaction between Sertoli and peritubular cells was carried out by placing a Sertoli cell-containing disc within the teflon cylinder (labelled S1 in Fig. 1) and a peritubular cell-containing disc on a culture dish (labelled S2 in Fig. 1). A teflon ring placed under $\mathrm{S} 2$ permitted access of culture medium to basal cell surfaces. The teflon cylinder containing S1 was placed over S2. S1 and S2 were separated by a distance of $2 \mathrm{~mm}$. Four grooves at the base of the teflon cylinder facilitated medium and gas equilibration (labelled $d$ in Fig. 1) and cell-cell paracrine interaction.

Apical/basal-compartmentalized fuid chamber. Sertolispermatogenic cells or Sertoli-peritubular cocultures were prepared on uncoated HATF discs. For Sertoli-peritubular cell cocultures, Sertoli cells were plated on confluent peritubular cell cultures (prepared by plating and then replating peritubular cells after $12-24 \mathrm{~h}$ intervals) both to establish continuous cell sheets and to reproduce cell relationships as found in vivo. The formation of continuous sheets of Sertoli-spermatogenic cells or Sertoli-peritubular cells was verified by measuring specific electrical resistance (see below). Equal volumes of culture medium $(0.5 \mathrm{ml})$ were maintained in apical and basal compartments to prevent the formation of concentration gradients. Fig. 1 shows the levels of culture medium attained in apical and basal compartments with respect to the cell-covered substrate (labelled $\mathrm{S}$ ). Grooves (labelled d) at the base of the teflon cylinder served for fluid equilibration within the basal compartment.

\section{Measurement of electrical resistance across cell sheets}

For studies on the sorting of Sertoli cell secretory protenn, specific electrical resistance to an applied current of $1.5 \mathrm{~V}$ was measured across cocultured cell sheets prepared on an uncoated microporous substrate to verify physical continuity of the cell layers. Peritubular cell cultures (cultured as shown in Fig. 2D), and cell-free uncoated microporous substrate placed in culture medium were used as controls. Electrical resistance was determined using $\mathrm{Ag}-\mathrm{AgCl}$ electrodes coupled to a digital multimeter (Simpson, Elgin, IL). Values were corrected for cell constant (distance between electrodes and area). Cell sheets (Sertoli cells cocultured with spermatogenic or peritubular cells) with calculated average electrical resistance of $0.7 \mu \mathrm{ohm} \mathrm{cm} \mathrm{c}^{-1}(n=14)$ observed 6 days after plating were used for protein-sorting studies. Control samples showed a calculated average electrical resistance of $0.018 \mu \mathrm{ohm} \mathrm{cm}^{-1}$ (peritubular cells alone, $n=8$ ) and $0.007 \mu \mathrm{ohm} \mathrm{cm} \mathrm{c}^{-1}$ (microporous substrate, $n=5$ ).

\section{$\left[{ }^{35}\right.$ S]methionine-labelling and two-dimensional polyacrylamide gel electrophoresis}

Cultured cells were labelled with $\left[{ }^{35} \mathrm{~S}\right]$ methionine (New England Nuclear, Boston, MA) as described (Kierszenbaum et al. 1986a), after 6 days of culture or coculture. Cells were placed in serum-free EMEM containing $0 \cdot 1$ of the usual concentration of methionine, supplemented with $250 \mu \mathrm{Ci} \mathrm{ml}^{-1}$ of $\left[{ }^{35} \mathrm{~S}\right]$ methionine. Cells were incubated for $18-24 \mathrm{~h}$, the radioactive medium was aspirated, the cells were then centrifuged $(15600 \mathrm{~g}, 5 \mathrm{~min})$, frozen $\left(-20^{\circ} \mathrm{C}\right)$, lyophilized and resuspended in lysis buffer (O'Farrell, 1975). Trichloroacetic acid-precipitable radioactivity in both the lyophilized culture medium and the reconstituted sample in lysis buffer was determined on glass microfibre filters following removal of non-incorporated radioactivity. Samples containing the same number of counts incorporated into proteins were analysed by two-dimensional polyacrylamide gel electrophoresis (2D-PAGE) and autoradiography as described (O'Farrell, 1975; Kierszenbaum et al. 1986a).

For radiolabelling studies using uncoated HATF microporous substrates, a volume of $0.5 \mathrm{ml}$ of radioactive medium

Fig. 2. Light microscopy of cultured and cocultured cells on different substrates. All illustrations represent 6-day-old cultures prepared from 20 - to 22 -day-old rats. A. Sertoli cells (SC) cultured on nylon coated with ECM material (provided by Dr H. Kleinman). $n$ is a cross-section of a nylon thread. I indicates typical lipid droplets in SC not observed in peritubular cells. The apical compartment is indicated. Maintaned in TKM (Tres et al. 1986). $\times 2520$. B. Peritubular cells (PTC) cultured on nylon mesh coated with ECM. $n$ indicates cross-sections of nylon thread. Maintained in serum-supplemented EMEM. $\times 2520$. C. Sertoli cells (SC) cultured on an uncoated HATF microporous filter. Arrows indicate basal cytoplasmic processes extendıng into the filter. Arrowheads indicate apically located spermatogenic cells. Lipid droplets $(l)$ are indicated in SC. Mantaned in TKM. $\times 2520$.

D. Peritubular cells (PTC) cultured on an uncoated HATF microporous filter. Note the absence of basal cytoplasmic processes and spaces between cells. Maintained in serumsupplemented EMEM. $\times 1000$. E. Peritubular cell (PTC)-Sertoli cell (SC) cocultured on an uncoated HATF microporous filter. The basal compartment is indicated as well as lipid droplets $(l)$ in SC. Maintained in serumsupplemented EMEM. $\times 1500$. 

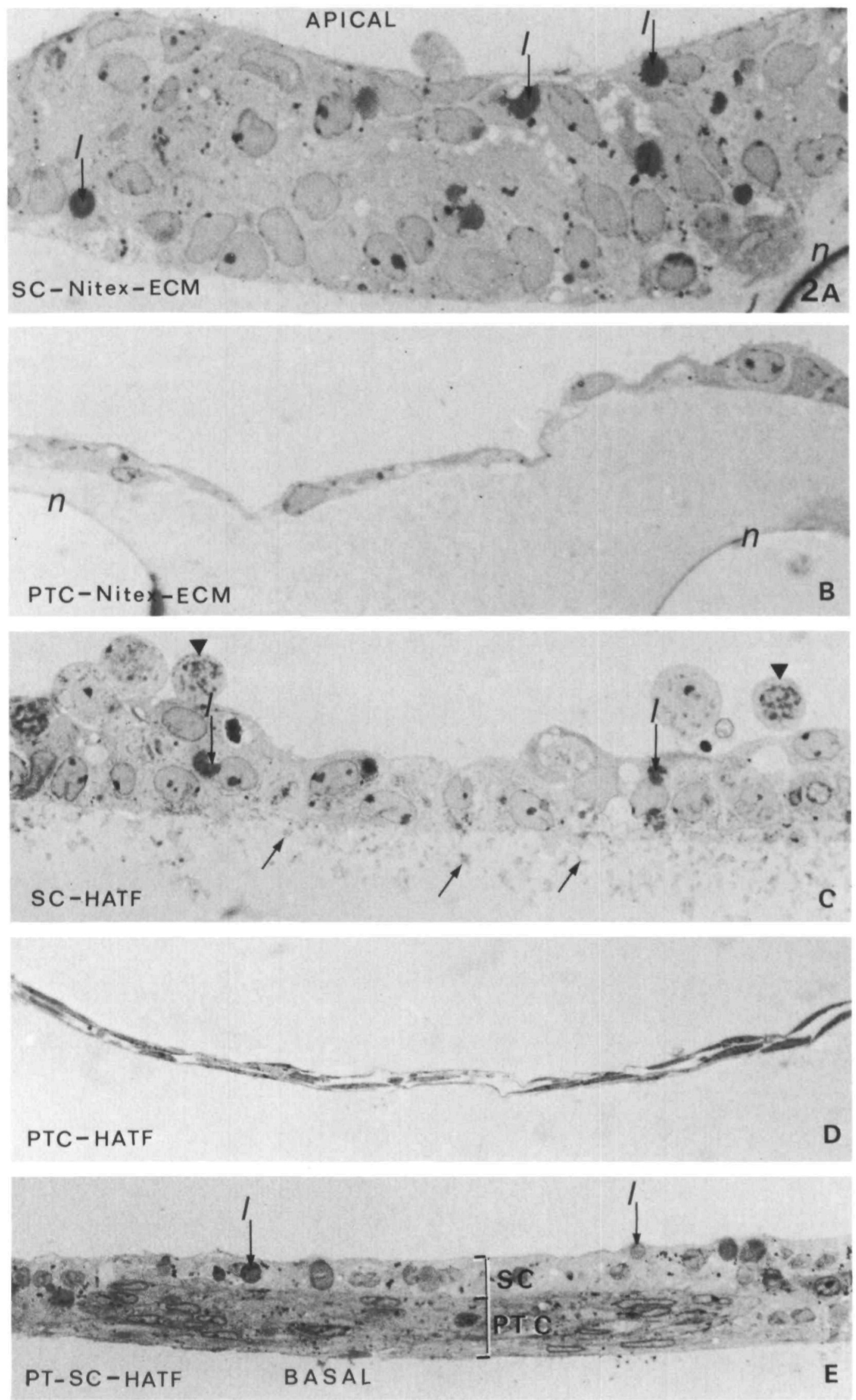
was used for upper and lower compartments. After radiolabelling, media from each compartment were carefully collected and processed as described above. Patterns of secretory proteins were similar whether $\left[{ }^{35} \mathrm{~S}\right]$ methionine was added to either or both apical/basal cell compartments.

\section{Detemination of comparative polypeptide radioactivity}

The position of the same radioactive polypeptide spot in a pair of gels containing proteins from apıcal and basal compartments was determined by overlapping the exposed autoradiogran $(n=6)$. Equivalent surface areas per polypeptide species in the gel were cut out and they contained protein extracted overnight with $0.1 \%$ sodium dodecyl sulphate at $40^{\circ} \mathrm{C}$. Scintillation fluid was added and cts $\min ^{-1}$ were determined by liquid scintillation spectrophotometry.

\section{Light and transmission electron microscopy and immunofuorescence microscopy}

Cells cultured on different substrates were fixed in $2.5 \%$ glutaraldehyde in $0.2 \mathrm{M}$-phosphate buffer ( $\mathrm{pH} 6.9$ ), postfixed in $2 \%$ osmium tetroxide in phosphate buffer, and embedded in epoxy resin according to standard procedure. Sections $0 \cdot 5-1 \mu \mathrm{m}$ thick made perpendicular to the substrate were stained with Toluidine Blue for light-microscopic examination. Thin sections were stained with uranyl acetate and lead citrate, and examined in a JEOL 100-CX transmission clectron microscope operated at $60 \mathrm{kV}$.

For indirect immunofluorescence, cultured cells were fixed in $3.7 \%$ formaldehyde in PBS for $15 \mathrm{~min}$ and embedded in Historesin (LKB, Bromma, Sweden). Plastic sections ( $1 \mu \mathrm{m}$ thick) were immunoreacted with monoclonal antibody IFP. $\mathrm{SC}$ as reported (Kierszenbaum et al. 1986b). IFP-SC recognizes a form of intermediate filament protein present in Sertoli cells that is absent in peritubular cells. Preparations were observed on a Leitz Ortholux II photomicroscope and images recorded on Kodak Tri-X film (Eastman Kodak, Rochester, NY).

\section{Results}

Topographic arrangement of cultured and cocultured cells on permeable substrates

Fig. 2 illustrates morphological aspects of Sertoli and peritubular cell cultures prepared on ECM-coated nylon and uncoated HATF microporous filter and grown for 6 days. The nylon mesh has large openings (about $100 \mu \mathrm{m}$ wide) requiring coating with ECM material to permit monolayer cell growth. We have used ECM derived from Engelbreth-Holm-Swarm tumours containing laminin, collagen type IV, heparan sulphate proteoglycan, nidogen and entactin as major components (Kleinman et al. 1986). Sertoli cells cultured on ECM-coated nylon (Fig. 2A) organized a multilayered structure devoid of spermatogenic cells but retaining typical nuclear and cytoplasmic features (Kierszenbaum \& Tres, 1981). Peritubular cells cultured on the same ECM-coated nylon substrate formed a characteristic monolayer (Fig. 2B), which required the presence of serum-supplemented EMEM for rapid growth. In contrast, Sertoli cell growth was effective in serum-free TKM. When Sertoli cells were cultured on HATF filters that did not require ECM coating because of their small pores (Fig. 2C) they were cuboid, as well as having two important polarity features: (1) the formation of multiple basal cytoplasmic processes projecting into the microporous HATF substrate; and (2) the apical distribution of meiotic prophase spermatocytes. These two features were not conspicuous in Sertoli cells cultured on ECM-coated nylon (compare with Fig. 1A).

Peritubular cells cultured on uncoated HATF discs organized discrete layers of flat cells lacking basal cytoplasmic processes (Fig. 2D). Fig. 2E illustrates a peritubular-Sertoli cell coculture on an uncoated HATF disc in which each cell population retains inherent structural features such as lipid-containing cuboidal Sertoli cells and flat peritubular cells. The multilayered organization of peritubular cells was due to two consecutive platings (at 12 -h intervals) to ensure a tight peritubular cell layer prior to the apical plating of trypsin-dissociated Sertoli cells.

Fig. 3A illustrates a rat peritubular-Sertoli cell coculture prepared on an uncoated HATF filter and immunoreacted with monoclonal antibody IFP-SC to identify Sertoli cells further by an intermediate filament protein not detected in peritubular cells (Kierszenbaum et al. 1986b). Sertoli cells show a diffuse cytoplasmic fluorescent pattern while peritubular cells are not immunoreactive. The intricate microporous organization of the HATF filter can be recognized by

Fig. 3. A. Localization of IFP-SC immunoreactive intermediate filament protenn in Sertoli cells (SC) not observed in subjacent peritubular cells (PTC). The fluorescent cellular signal tends to be weak in plasticembedded and relatively thin $(1 \mu \mathrm{m})$ sectioned specimens. HATF is the microporous substrate. $\times 1575$. B. Lightmicroscopic view of same specimen as in A showing multilayered peritubular cells (PTC) and a layer of cuboidal Sertoli cells (SC) with typical lipid (l) droplet. Maintaıned in coculture for 6 days in serum-supplemented EMEM. $\times 3024$. C. Same specimen as in B showing Sertoli cells (SC) and peritubular cells (PTC) cocultured on an uncoated HATF microporous filter. Arrowheads indicate densities corresponding to occluding junctions between adjacent Sertoli cells and between Sertoli cell basal cytoplasmic processes. Note the space between SC and PTC (containing ectoplasmic microfilaments). Cultured in serum-supplemented medium for 6 days. $\times 6000$. D. Basal region of Sertoli cells (SC) cultured on an uncoated HATF microporous filter. The arrows indicate adhesion plaques in basal cytoplasmic processes projecting into HATF. The arrowheads indicate a lateral occluding junction. Cultured in TKM for 6 days. $\times 18500$. 
impregnation with rhodamine fluorochrome conjugated to goat anti-mouse immunoglobulins used as second antibody during immunostaining. Fig. $3 \mathrm{~B}$ is a bright-field microscopic view of the same coculture for comparison with Fig. $3 \mathrm{~A}$.

A transmission electron-microscopic study of Sertoli-peritubular (Fig. 3C) and Sertoli-spermato- genic cell cocultures (Figs 3D, 4A-C) prepared on uncoated microporous substrate demonstrates the following structural features: (1) adjacent Sertoli cclls display tight junctions that occlude the intercellular spaces, as verified by electrical resistance studics (Fig. 3C); (2) junctional complexes were not detected between Sertoli and subjacent peritubular cells or
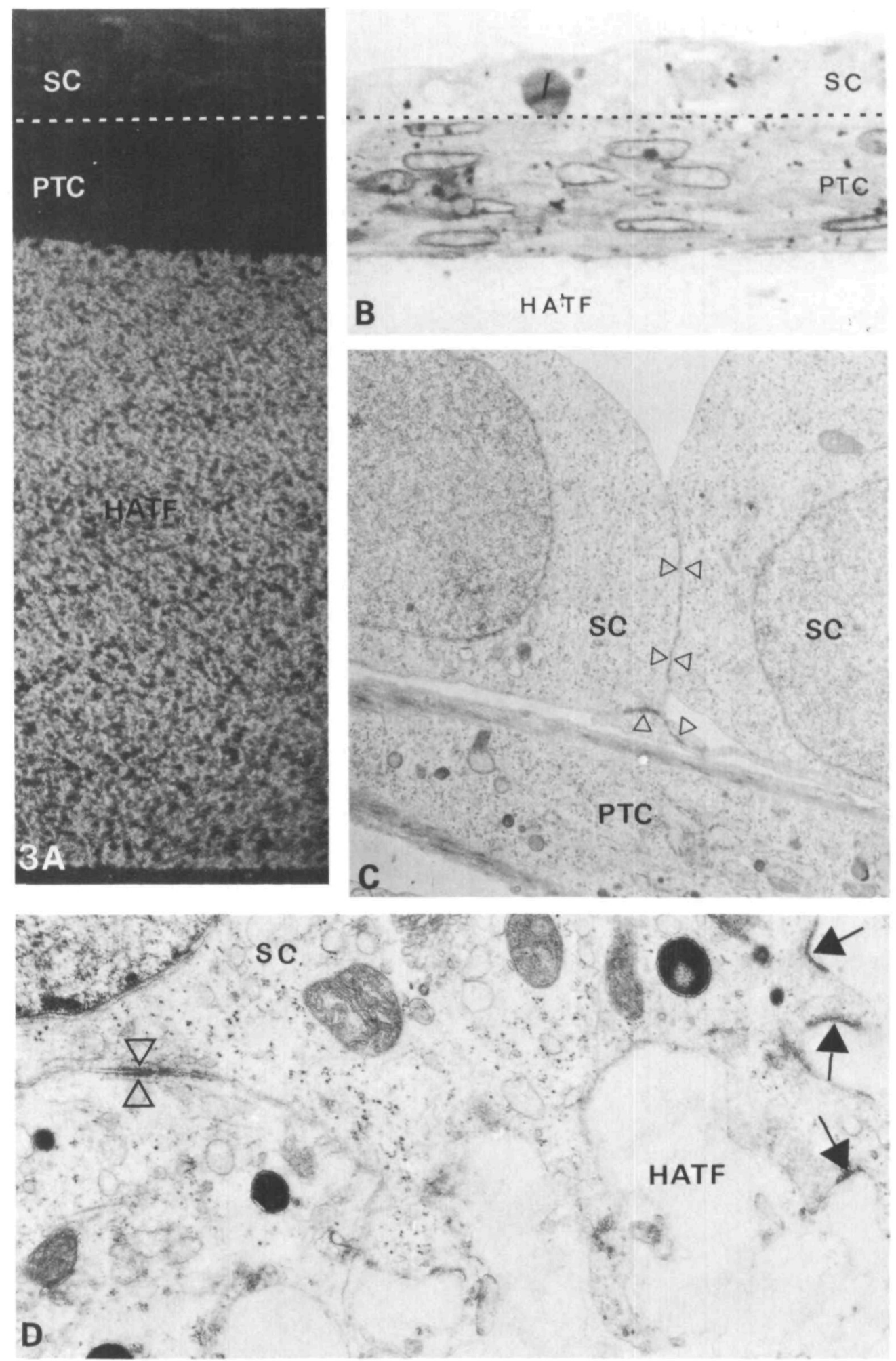
among peritubular cells (Fig. 3C); (3) in the absence of subjacent peritubular cells, Sertoli cells project multiple branching cytoplasmic processes into the microporous substrate and display typical adhesion plaques (Fig. 3D); (4) in Sertoli-spermatogenic cell cocultures, the apical cell surfaces of Sertoli cells are associated with meiotic prophase spermatocytes (Fig. 4A) whereas spermatogonia are more basally located and surrounded by Sertoli cells (Fig. 4D); and (5) a characteristic ectoplasmic differentiation of Sertoli cells in vivo (consisting of endoplasmic reticulum cisternae and bundles of microfilaments) is also observed in culture, facing an adjacent Sertoli cell lateral surface with features characteristic of endocytic activity (Fig. 4C).

\section{Protein secretory patterns of Sertoli and peritubular cells cultured on ECM-coated nylon mesh and uncoated HATF filters}

Newly synthesized proteins of Sertoli and peritubular cells were analysed to determine whether different substrates can modify protein secretory patterns. Fig. $5 A, B$ shows that Sertoli and peritubular cells cultured on ECM-coated nylon and uncoated HATF filters secreted essentially similar protein patterns, although some differences in the labelling intensity of some proteins could be detected. Sertoli cells cultured on either ECM-coated nylon or uncoated HATF filters secreted transferrin, S70 and S45-S35 heterodimeric protein as well as SP1 and SP2, which are proteins also secreted by peritubular cells (Kierszenbaum et al. 1986a). SP1 and a group of less-acidic proteins in the $M_{r}$ range $43000-30000$ appeared more prominent in the medium of Sertoli cells cultured on uncoated microporous substrate than on ECM-coated nylon mesh (Fig. 5A). This difference can be attributed either to possible variations between different cultures or, most likely, to an effect of the substrate on Sertoli cell secretory activity.

As reported (Kierszenbaum et al. 1986a), cultured peritubular cells secreted a characteristic group of high molecular weight proteins (not found in cultured Sertoli cells) as well as SP1 and SP2 (Fig. 5B). Sertoli cell secretory proteins S70 and S45-S35 heterodimeric protein were not detected in the radiolabelled medium of peritubular cells as expected (Kierszenbaum et al. 1986a).

Additional cell-cell interacting experiments using the one-fluid compartment (illustrated in Fig. 1, part 1) resulted in combined cell-specific secretory protein patterns (not shown).
Sorting of secretory proteins released into apical and basal compartments of cocultured Sertoli cell and peritubular cells

Our next approach was to evaluate the vectorial sorting of transferrin, S70 and S45-S35 heterodimeric protein using Sertoli and peritubular cells cocultured on uncoated HATF microporous substrates. Sertoli cells formed a continuous cell sheet displaying electrical resistance attributed to the formation of lateral occluding junctions.

Fig. 5C shows a compartmentalized distribution of Sertoli cell secretory proteins: while comparably less transferrin and S70 were found in the basal compartment, little or no S45-S35 heterodimeric protein was detected. Although SP1 and SP2 were found in both the basal and apical cell compartments, the radiolabelling of SP1 was slightly higher in samples from the basal compartment. In addition, more transferrin appears in the apical compartment than in the basal compartment. High molecular weight secretory proteins secreted by peritubular cells appeared more apically sorted (Fig. 5C). Similar results were obtained with Sertoli cells alone that were cultured on uncoated HATF microporous substrate as shown in Table 1. Table 1 provides cts $\min ^{-1}$ values that confirm subjective estimates of $\left[{ }^{35} \mathrm{~S}\right]$ methionine-labelling intensity of similar polypeptides detected in upper and lower medium compartments of cultured Sertoli and peritubular cells, and cocultured Sertoli and peritubular cells resolved by electrophoresis and autoradiography.

The study of sorted proteins secreted by peritubular cells was hampered by the 'leaky' nature of these cultured cells as inferred from structural studies

Fig. 4. A. Apical region of Sertoli-spermatogenic cells cocultured on uncoated HATF microporous filter. Meiotic prophase spermatocytes (leptotene-zygotene) $(s p c)$ are associated with Sertoli cell $(S c)$ apical surfaces. The arrows indicate Sertoli cell cytoplasmic processes surrounding one of the spermatocytes. Cultured in TKM for 6 days. $\times 16000$. B. Rat Sertoli-spermatogenic cell coculture prepared and maintained as in A. A spermatogonium type A $(s p g)$ is enbedded in a Sertoli cell ( $S c$, arrows). HATF identifies the microporous substrate into which SC cytoplasmic processes project (crossed filled arrows). In the apical region of the coculture, a bracket denotes

overlapping cytoplasmic parts of two adjacent SC. An open crossed arrow indicates a characteristic long and narrow SC apical cytoplasmic process. $\times 10400$. C. Rat

Sertoli-spermatogenic cell coculture prepared and maintained as in A. Lateral intercellular face between two adjacent Sertoli cells identified by two horizontal arrows at the top of the figure. The ectoplasmic portion of the cell at the right depicts endoplasmic reticulum cisternae (arrowheads) and bundles of microfilaments (lines). The cell surface of the cell at the left depicts coated pits and vesicles (arrows) as an indication of receptor-mediated endocytic activity. $\times 47500$. 

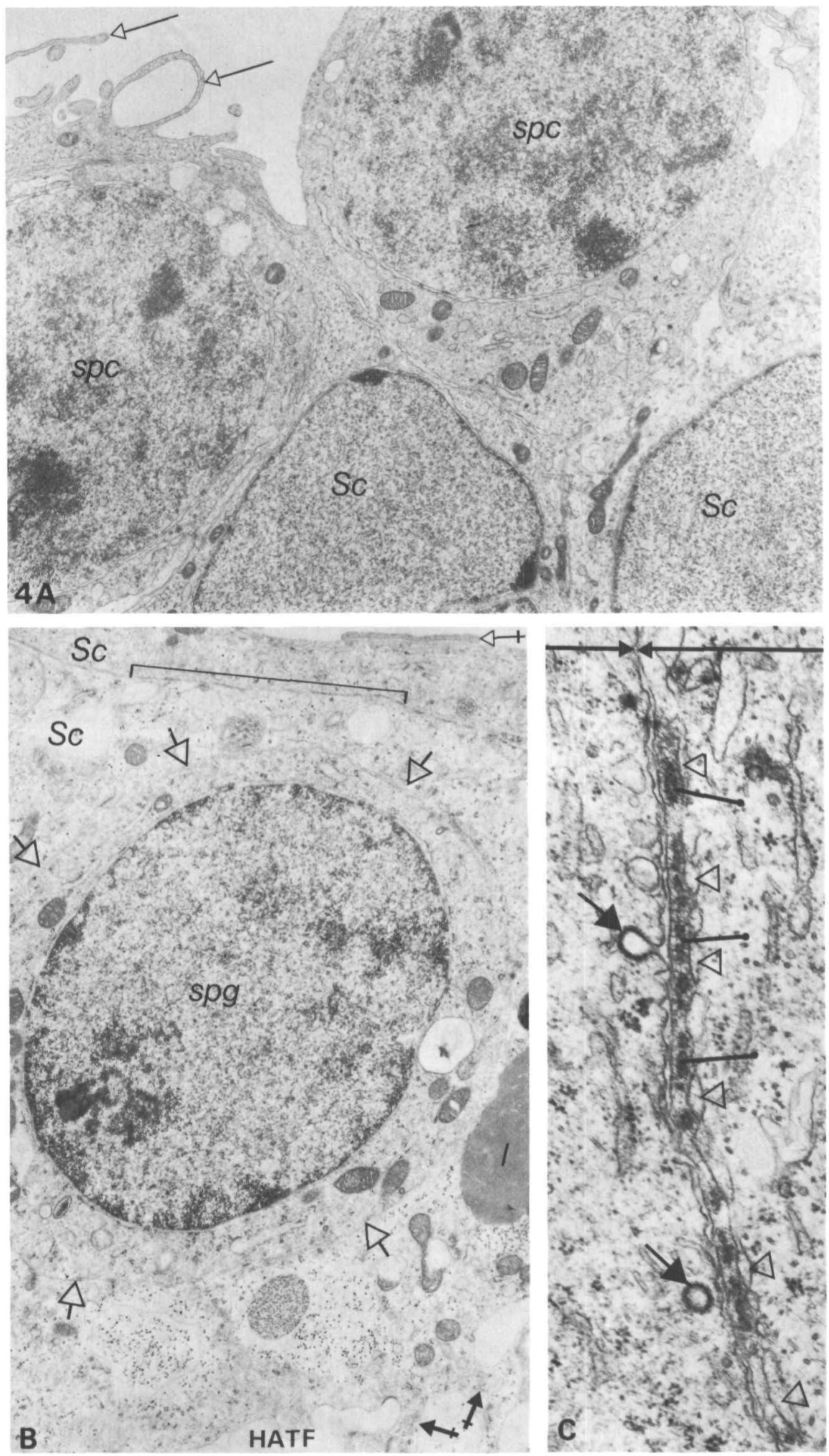


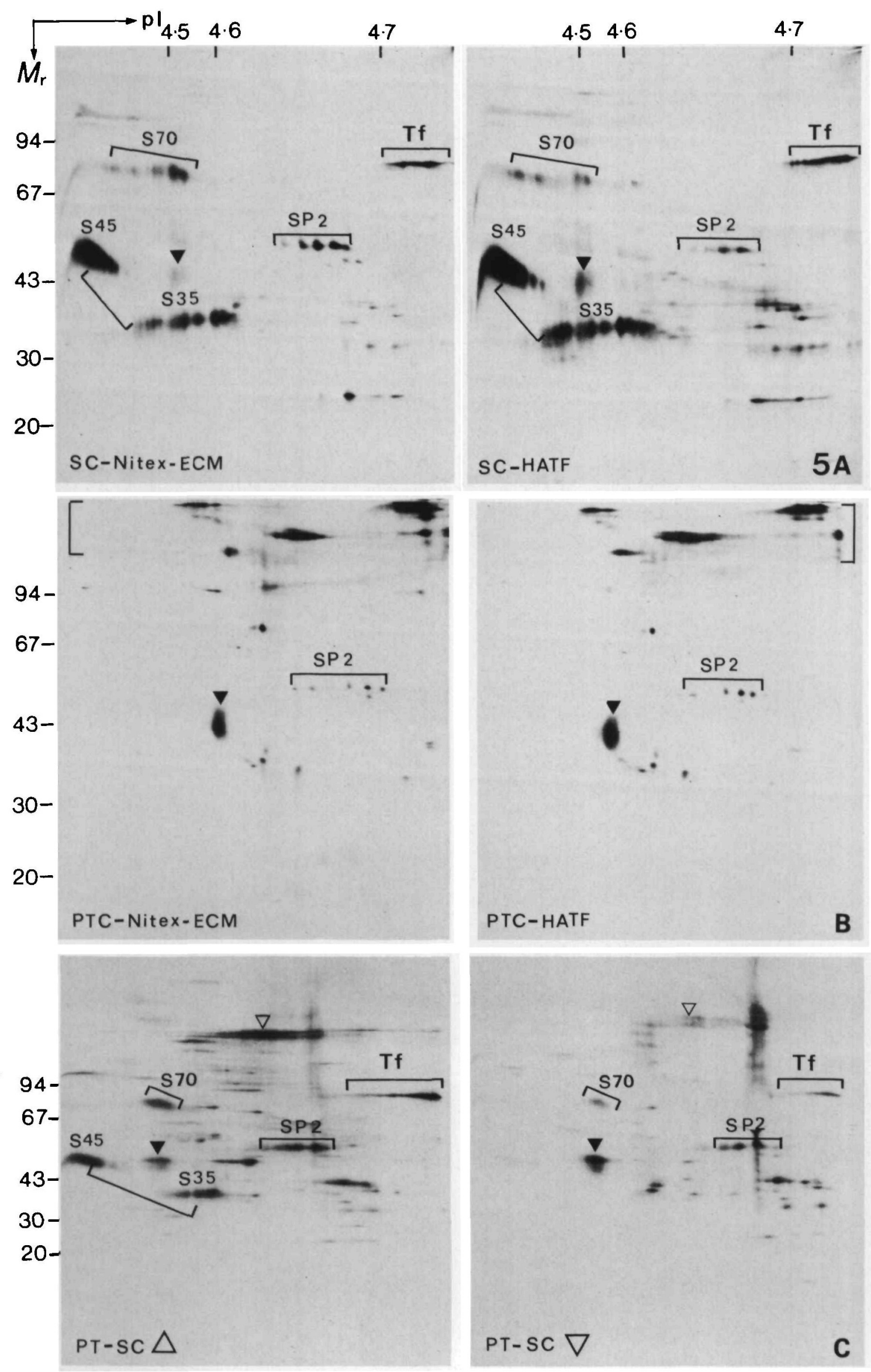


Table 1. Comparative radioactivity of ${ }^{35}$ S]methionine-labelled polypeptides

\begin{tabular}{|c|c|c|c|c|c|c|}
\hline \multirow[b]{2}{*}{ Polypeptide } & \multicolumn{3}{|c|}{ Apical compartment } & \multicolumn{3}{|c|}{ Basal compartment } \\
\hline & $\mathrm{SC}$ & PTC & S-PTC & $\mathrm{SC}$ & PTC & S.PTC \\
\hline Transferrin & $286(3 \cdot 4)$ & $\mathrm{BG}$ & $245(2.9)$ & 84 & BG & 84 \\
\hline S70 & $192(2 \cdot 5)$ & BG & $161(2 \cdot 4)$ & 78 & $\mathrm{BC}$ & 66 \\
\hline SP1 & 230 & 168 & 144 & 217 & 131 & $368(2 \cdot 5)$ \\
\hline \multicolumn{7}{|c|}{$\begin{array}{l}\text { Cts } \min ^{-1} \text { valucs for equivalent surface area of radiolabelled spots were determined within pairs of electrophoretic protein maps and } \\
\text { corrected for background (BG). Fold increase of the other spot was calculated and indicated in parenthesis. Cells were cultured on } \\
\text { uncoated HATF substrate. SC, Sertoli cell cocultures containing about } 30 \% \text { spermatogonia and meiotic prophase spermatocytes; } \\
\text { PTC, peritubular cell cultures; S-PTC, Sertoli-peritubular cell cocultures. }\end{array}$} \\
\hline
\end{tabular}

(Fig. 1D) and by measurement of electrical resistance (see Materials and methods).

\section{Discussion}

We have examined the topographic arrangement of cultured rat Sertoli and peritubular cells, and cocultured Sertoli-peritubular cells on two permeable substrates differing in their porous openings and coating with ECM material. These studies were conducted for determining the organization of cell sheets on different permeable substrates for use in automated cell perifusion studies (Tres \& Kierszenbaum, 1986; Kierszenbaum \& Tres, 1987).

We have determined possible contributions of these substrates to the pattern of cell-specific secretory proteins and evaluated the vectorial sorting of Sertoli cell secretory proteins under conditions that permit separation of apical and basal cell compartments. We provide evidence supporting vectorial protein secretory

Fig. 5. Two-dimensional gel electrophoresis and autoradiography of secretory proteins from Sertoli (SC) and peritubular cells (PTC) cultured or cocultured on the indicated substrates. Numbers at the left are $M_{\mathrm{r}} \times 10^{-3}$. Numbers on top are pI. Tf, transferrin. The arrowhead, indicating SP1, and the bracket showing SP2, denote two secretory protein that are common products of Sertoli and peritubular cells (Kierszenbaum et al. 1986a). Vertical brackets in B indicate high $M_{\mathrm{r}}$ proteins secreted by PTC that are not observed in SC samples. The arrowheads in $\mathrm{C}$ indicate a high $M_{\mathrm{r}}$ protein secreted by PTC in

Sertoli-peritubular cell cocultures (PT-SC). The position of Sertoli cell-specific proteins S70 and S45-S35 heterodimeric protem is indicated. Autoradiograms of SC samples (A) represent $600000 \mathrm{cts} \mathrm{min}^{-1}$ and 1 week of exposure. Autoradiograms of PTC samples (B) represent $300000 \mathrm{cts} \mathrm{min}^{-1}$ and 1 week of exposure. Autoradiograms of PT-SC coculture samples (C, prepared on uncoated HATF microporous substrate) represent $700000 \mathrm{cts} \mathrm{min}^{-1}$ and 2 weeks of exposure. In $\mathrm{C}$, an arrowhead pointing upwards indicates medium sample from the apical compartment; an arrowhead pointing downwards indicates medium sample from the basal compartment. functions that strengthen the polar organization of Sertoli cells in vitro.

Polarity appears as a phenotypic property of Sertoli cells, since peritubular cells: (1) do not display significant release of vectorial protein; (2) do not modify the sorting of Sertoli cell newly synthesized secretory proteins; (3) do not develop basal cytoplasmic processes as Sertoli cells do; (4) do not form tight junctions (Dym \& Fawcett, 1970); and (5) do not offer electrical resistance to an applied electrical current.

Two aspects in this paper are of interest: (1) the culture conditions that permit vectorial functions, and (2) the significance of protein secretory sorting.

We have shown that while Sertoli cells cultured on ECM-coated nylon discs become multilayered, they neither develop multiple basal cytoplasmic processes nor retain their association with spermatogenic cells that become depleted from the cultures during medium replenishment. However, Sertoli cells cultured on uncoated HATF discs create more specialized ccll surfaces by developing abundant basal cytoplasmic processes and providing apical attachment sites for spermatogenic cells. The development of basal cytoplasmic processes depends on the microporous nature of the HATF substrate and not on the absence of ECM material, since: (1) similar cytoplasmic processes have been observed in Sertoli cells cultured on ECM-coated HATF filter (Byers et al. 1986; Djakiew et al. 1986); (2) basal cytoplasmic processes are not observed in Sertoli cells cultured on ECM-coated nylon; and (3) in vivo, the basal surface of rat Sertoli cell in contact with basal lamina is not characterized by conspicuous cytoplasmic processes (Dym \& Fawcett, 1970).

We have not been able to confirm a favourable effect of ECM on the development of spermatogenic cells in coculture with Sertoli cells as reported (Hadley et al. 1985). We have observed prevalent multilayered Sertoli cells but very few spermatogenic cells in cultures prepared on ECM similar to that used by Hadley et al. (1985). It is likely that a few spermatogenic cells may remain structurally viable but the demonstration of development in vitro requires more stringent parameters such as the progression of $\left[{ }^{3} \mathrm{H}\right]$ thymidine- 
labelled preleptotene spermatocytes in their development (Tres \& Kierszenbaum, 1983), and the differential expression of somatic histone $\mathrm{H} 2 \mathrm{~B}$ and testisspecific histone TH2B genes in premeiotic and meiotic spermatogenic cells (Kim et al. 1987). Further studies should demonstrate whether other types of ECM exert a favourable effect on spermatogenic cell long-term viability and differentiation in culture. The finding that Sertoli cells cultured for 6 days on ECM-coated nylon mesh with wide openings are essentially devoid of spermatogenic cells is of practical significance because this substrate permits functional studies of Sertoli cell populations only.

The compartmentalization of apical and basal cell spaces by cultured Sertoli cells and cocultured peritubular-Sertoli cells is largely dependent on the establishment of continuous cell monolayers and complete sealing of intercellular spaces for maintaining concentration gradients between the compartments they separate. The formation of continuous Sertoli cell sheets is readily apparent by the electron-microscopic demonstration of basal occluding junctions between adjacent Sertoli cells that restrict access to electron-opaque tracers (Hadley et al. 1985; Byers et al. 1986) and by the establishment of electrical resistance across the cell sheet. However, peritubular cells in culture form a 'leaky' monolayer or bilayer with low electrical resistance. Therefore, it is not surprising that SP1 and SP2 can be found in both basal and apical compartments (Table 1). The inherent permeable nature of peritubular cell layers was not eliminated by double-plating trypsin-dissociated peritubular cells (previously prepared in $75 \mathrm{~cm}^{2}$ tissue culture flasks) on uncoated HATF discs to create a multilayered peritubular cell growth. This approach was not feasible on ECMcoated HATF because of the tendency of peritubular cells to form spaced domes (not shown).

Despite the establishment of multilayered peritubular cell cultures on which Sertoli cells were plated, we have found that the concentration gradient of transferrin and $S 70$ remained unchanged when compared with Sertoli cells cocultured with spermatogenic cells (Table 1). An exception was SP1, which showed a $2 \cdot 5$-fold increase in the basal compartment when compared with the apical compartment. In addition, high $M_{\mathrm{r}}$ proteins secreted by peritubular cells in coculture with Sertoli cells are not abundant when compared with peritubular cell cultures. Whether high $M_{\mathrm{r}}$ proteins are synthesized and secreted but bind to cell surfaces to organize a basal lamina-like structure (Tung et al. 1985) remains to be studied.

Another interesting aspect of this work is the unidirectional and bidirectional sorting of Sertoli cell newly synthesized secretory proteins, because of their possible role in spermatogenesis. Our studies show the bidirectional release of transferrin and S70 and the unidirectional apical release of S45-S35 heterodimeric protein. We confirm a previous report showing that cultured Sertoli cells prepared on uncoated-HATF microporous substrates secrete transferrin bidirectionally (Janecki \& Steinberger, 1986, 1987). The functional significance of the bidirectional release of transferrin by Sertoli cells in culture and its possible relation with the in vivo condition are difficult to correlate because of the enhancement of transferrin gene activity and expression by Sertoli cells in culture (Lee et al. 1986).

The observation that $\mathrm{S} 70$ and $\mathrm{S} 45-\mathrm{S} 35$ heterodimeric proteins are apically released while transferrin release is bidirectional confirms the polar nature of Sertoli and peritubular-Sertoli cell cocultures. Previous studies have shown the bipolar secretion of androgen-binding protein and transferrin by Sertoli cells cultured in a two-compartment culture chamber (Janecki \& Steinberger, 1986, 1987) that is essentially similar to our cultivation teflon chamber. Findings reported in this paper and by Danahey et al. (1986) are the first to show a selective unidirectional sorting of Sertoli cell secretory proteins. In addition, our studies demonstrate that spermatogenic and peritubular cells cocultured with Sertoli cells contribute little to the vectorial sorting of Sertoli cell secretory proteins. Because the synthesis of S70 and S45-S35 heterodimeric protein is associated with spermiogenesis (Shabanowitz et al. 1986; Kierszenbaum et al. 1987), the vectorial apical release of these proteins by Sertoli cells may represent an in vitro correlate reflecting utilization by adluminally located spermatids in the seminiferous tubule.

In summary, we have presented morphological and electrophoretic data that confirm Sertoli cell polarity and protein sorting in vitro. In agreement with reports from other laboratories (Hadley et al. 1985; Byers et al. 1986; Djakiew et al. 1986; Janecki \& Steinberger, 1986, 1987), Sertoli-spermatogenic cell cocultures (Kierszenbaum \& Tres, 1987) and peritubular-Sertoli cell cocultures (prepared as described in this paper) provide experimental systems that approach the situation in vivo by mimicking the basic organization of the seminiferous tubule, including the physiological tubular barrier. These physiological aspects are being explored by automated cell perifusion using Sertolispermatogenic cell cocultures prepared on peritubular cell monolayers.

We thank Ms Liu Ping for processing specimens for light microscopy and immunofluorescence, and Drs Eric P. Smith and Robert B. Shabanowitz for their participation in earlier phases of this project. Dr Hiroshi Ueda is recipient of a Fogarty International Fellowship (1F05 TW0381). This work was supported by NIH grant HD11884. 


\section{References}

Byers, S. W., Hadley, M. A., Duakiew, D. \& Dym, M. (1986). Growth and characterization of polarized monolayers of epididymal epithelial cells and Sertoli cells in dual environment culture chambers. $\mathcal{J}$. Androl. 7, $59-68$.

Cereijido, M., Robbins, E. S., Dolan, W. J., Rotunno, C. A. \& Sabatini, D. (1978). Polarized monolayers formed by epithelial cells on a permeable and translucent support. F. Cell Brol. 77, 853-880.

Danahey, D. G., Letscher, R. L. \& DePhilip, R. M. (1986). Evidence for the establishment of functional polarity by Sertoli cells in vitro. $\%$. Cell Biol. 103, 484a.

DePhilip, R. M. \& Kierszenbaum, A. L. (1982).

Hormonal regulation of protein synthesis, secretion, and phosphorylation in cultured rat Sertoli cells. Proc. natn. Acad. Sci. U.S.A. 79, 6551-6555.

Djakiew, D., Hadley, M. A., Byers, S. W. \& Dym, M. (1986). Transferrm-mediated transcellular transport of ${ }^{59} \mathrm{Fe}$ across confluent epithelial sheets of Sertoli cells grown in bicameral cell culture chambers. F. Androl. 7, 355-366.

Dym, M. \& FawcetT, D. W. (1970). The blood-test1s barrier in the rat and the physiological compartmentalization of the seminiferous epithelium. Biol. Reprod. 3, 308-326.

Hadley, M. A., Byers, S. W., Suarez-Quian, C. A., Kleinman, H. K. \& DYM, M. (1985). Extracellular matrix regulates Sertoli cell differentiation, testıcular cord formation, and germ cell development in vitro. $\mathcal{F}$. Cell Biol. 101, 1511-1522.

Hutson, J. C. (1983). Metabolic cooperation between Sertoli cells and peritubular cells in culture.

Endocrinology 112, 1375-1381.

Hutson, J. C. \& Stocco, D. M. (1981). Peritubular cell influence on the efficiency of androgen-binding protein secretion by Sertoli cells in culture. Endocrinology 108, 1362-1368.

Janecki, A. \& Steinberger, A. (1986). Polarized Sertoli cell functions in a new two-compartment culture system. 7. Androl. 7, 69-71.

JANECKI, A. \& STEINBERGER, A. (1987). Bipolar secretion of androgen-binding protein and transferrin by Sertoli cells cultured in a two-compartment culture chamber. Endocrinology 120, 291-298.

Kierszenbaum, A. L., Abdullah, M., Ueda, H. \& TRES, L. L. (1987). Spermatogenesis in vitro: searching for in vizo correlates. In Regulation of Ovarian and Testicular Function (ed. V. B. Mahesh, E. Anderson \& D. Dhindsa), pp. 535-560. New York: Plenum Press.

Kierszenbaum, A. L., Crowell, J. A., Shabanowitz, R. B., DePhilip, R. M. \& Tres, L. L. (1986a). Protein secretory patterns of rat Sertoli and peritubular cells are influenced by culture conditions. Biol. Reprod. 35, 239-251.

Kierszenbaum, A. L., Crowell, J. A., Shabanowitz, R. M., Sмith, E. P., Spruill, W. A. \& Tres, L. L. (1986b). A monoclonal antibody recognızes a form of intermediate filament protein in rat Sertoli cells that is not present in semıniferous peritubular cells. Biol. Reprod. 35, 227-238.

Kierszenbaum, A. L. \& Tres, L. L. (1981). The structural and functional cycle of Sertoli cells in culture. In Bioregulators of Reproduction (ed. G. Jagiello \& H. J. Vogel), pp. 207-228. New York: Academic Press.

Kierszenbaum, A. L. \& Tres, L. L. (1987). An automated perifusion system for the study of rat spermatogenesis in vilmo. Alln. NY Acad. Sci. (in press).

Kim, Y.-J., Huang, I., Tres, L. L., Kierszenbaum, A. L. \& CHAE, C.-B. (1987). Molecular cloning and differential expression of somatic and testis-specific 1I2B histone genes during rat spermatogenesis. Dev/ Biol. 124, 23-34.

Kissinger, C., Skinner, M. K. \& Griswold, M. D. (1982). Analysis of Sertoli cell-secreted proteins by twodimensional gel electrophoresis. Biol. Repiod. 27, 233-240.

Kleinman, H. K., McGarvey, M. L., Hassell, J. R., Star, V. L., Cannon, F. B., Laurie, G. W. \& Martin, G. R. (1986). Basement membrane complexes with biological activity. Biochemistry 25, 312-318.

Lee, N. T., Chae, C.-B. \& Kierszenbaum, A. L. (1986). Contrasting levels of transferrin gene activity in cultured rat Sertoli cells and intact seminiferous tubules. Proc. natn. Acad. Sci. U.S.A. 83, 8177-8181.

Misfeldt, D. S., Hamamoto, S. T. \& Pitelka, D. R. (1976). Transepithelial transport in cell culture. Proc. natn. Acad. Sci. U.S.A. 73, 1212-1216.

O'FARRELl, P. H. (1975). High resolution two-dimensional gel electrophoresis of proteins. 7. biol. (hem. 250, 4007-4021.

Setchell, B. P. (1978). Fluid secretion and the entry of substances into the tubules. In The Mammalian Testis, pp. 233-284. Ithaca: Cornell University Press.

Shabanowitz, R. B., DePhilip, R. M., Crowell, J. A., Tres, L. L. \& Kierszenbaum, A. L. (1986). Temporal appearance and cyclic behavior of Sertoli cell-specific secretory protems during the development of the rat seminiferous tubule. Biol. Reprod. 35, 745-760.

Skinner, M. K. \& FrITZ, I. B. (1985). Testicular peritubular cells secrete a protein under androgen control that modulates Sertoli cell function. Proc. natn. Acad. Sci. U.S.A. 82, 114-118.

Skinner, M., Tung, P. S. \& Fritz, I. B. (1985). Cooperativity between Sertoli cells and testicular peritubular cells in the production and deposition of extracellular matrix components. $\%$. Cell Biol. 100, 941-947.

Tres, L. L. \& Kierszenbaum, A. L. (1983). Viability of rat spermatogenic cells in zitro is facilitated by their coculture with Sertoli cells in serum-free, hormonesupplemented medium. Proc. naln. Acad. ScI. LiS.A. 80, 3377-3381. 
Tres, L. L. \& Kierszenbaum, A. L. (1986). Automated perifusion of rat Sertoli-spermatogenic cell cocultures. $\mathcal{F}$. Cell Biol. 103, 483a.

Tres, L. L., Smith, E. P., VAN Wyk, J. J. \&

Kierszenbaum, A. L. (1986). Immunoreactive sites and accumulation of somatomedin- $C$ in rat

Sertoli-spermatogenic cell co-cultures. Expl Cell Res. $162,33-50$.
TUNG, P. S. \& FrITZ, I. B. (1980). Interactions of Sertoli cells with myoid cells in vilro. Biol. Reprod. 23, 207-217. Tung, P. S., Skinner, M. \& Fritz, I. B. (1985). Cooperativity between Sertoli cells and peritubular cells in the formation of the basal lamina in the seminiferous tubule. Ann. N.Y. Acad. Sct. 438, 435-446.

(Received 14 September 1987 - Accepted 20 October 1987) 